EPJ Web of Conferences 92,02105 (2015)

DOI: $10.1051 /$ epjconf/ 20159202105

(C) Owned by the authors, published by EDP Sciences, 2015

\title{
Experimental study of effect of stenosis geometry on flow parameters
}

\author{
Ondřej Veselý ${ }^{1, a}$ Ludmila Nováková $^{1}$ and Josef Adamec $^{1}$ \\ ${ }^{1}$ CTU in Prague, Faculty of Mechanical Engineering, Department of Fluid Dynamics and Thermodynamics, 16607 \\ Prague 6, Czech Republic
}

\begin{abstract}
A stenosis is a narrowing in a tubular organ or structure. In medicine, vessel stenosis poses health risks for people. In this work, experimental investigations of pressure loss coefficient for varying stenosis eccentricity and shape were performed. Five models of different geometry were studied; all models were stenosis of $75 \%$ area reduction. The flow conditions approximate physiological flow. The measuring range of Reynolds number was from 130 to 2730 , measured values of pressure loss coefficient were from 12 to 20 . The steady experimental results indicated that static pressure loss coefficient is affected by the shape of stenosis, but it was affected more significantly by the eccentricity. Visualization experiments have been performed in Polycarbonate models.
\end{abstract}

\section{Introduction}

Stenosis is defined as a narrowing of a vessel. It is capable of causing pressure losses as blood flows through a vessel. The change in pressure has implications for the functioning of the body's systems. For example carotid artery stenosis can give rise to an ischemic attack [1]. Generally the stenoses are characteristic feature of the atherosclerosis.

The relationship between the occurrence of the atherosclerosis and local hemodynamic flow characteristics has been highlighted by many studies [2], [3], [4]. Both technical complications of required experiments and ethical limitations of humans studies have caused the transfer of the research from the medical field to the field of technology and the experiments are carried out more frequently in laboratories with utilization of technically advanced methods. Although blood in non-Newtonian fluid, for certain experiments conditions (e.g. for arteries with a larger diameter than $0.5 \mathrm{~mm}$ ) the non-Newtonian characteristics can be neglected and the use of Newtonian fluid is sufficient [5], [6]. The range of cardiovascular formations analysed in laboratory conditions most frequently comprises vessel narrowing with a variable degree of lumen reduction, i.e. stenosis. Attention is generally focused on models with a solid [7], [8], [9] as well as elastic wall [10], [11].

Young [12] developed a correlation between pressure loss across stenosis and stenosis dimension:

$$
\frac{\Delta p}{\rho U_{0}^{2}}=\frac{K_{v}}{R e}+\frac{K_{t}}{2}\left(\frac{A_{0}}{A_{1}}-1\right)^{2},
$$

where $\Delta p$ is the pressure loss, $\rho$ is the density of blood, $\mathrm{Re}$ is the Reynolds number, $\mathrm{A}_{0}$ is the crosssectional area of unobstructed vessel, $A_{1}$ is cross-sectional area of the obstructed vessel, average value of $\mathrm{K}_{\mathrm{t}}$ for the blunt plug stenosis is 1.52 and $\mathrm{K}_{\mathrm{v}}$ was given by the equation [12]:

$$
K_{v}=32 \frac{0.83 L_{s}+1.64 D_{1}}{D}\left(\frac{A_{0}}{A_{1}}\right)^{2},
$$

where $\mathrm{L}_{\mathrm{s}}$ is the stenosis length, $\mathrm{D}$ is the diameter of unobstructed vessel and $D_{1}$ is diameter of obstructed vessel.

In this work five models were studied; three symmetric stenoses with different narrowing angle and two eccentric with different geometry of cross-section of obstructed part of model. All models were stenosis with $75 \%$ area reduction.

For experiment, the friction pressure loss was neglected and the local pressure loss is given by the equation:

$$
\Delta p_{l}=\xi \rho \frac{v^{2}}{2},
$$

where $\Delta p_{1}$ is the local pressure loss, $\xi$ is the local pressure loss coefficient and $\mathrm{v}$ is the flow velocity in the unobstructed part of model.

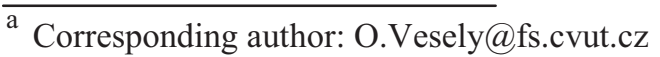




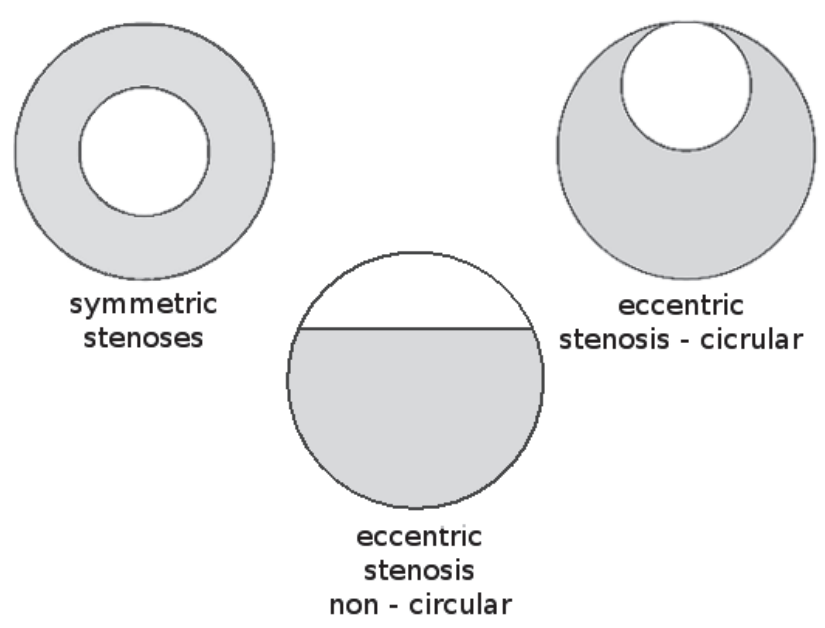

Figure 1. Cross-sections of the models used in the experiment.

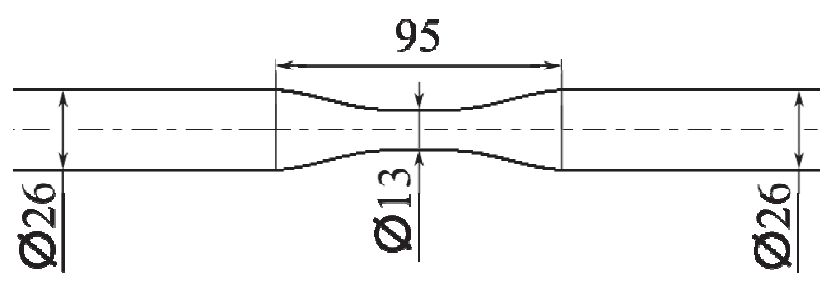

Figure 2. Symmetric stenoses number 1, narrowing angle $15^{\circ}$.

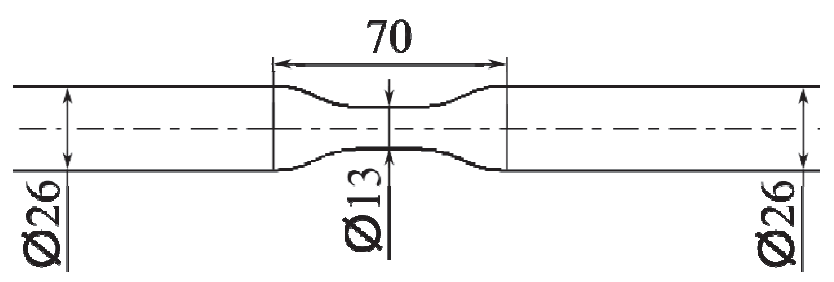

Figure 3. Symmetric stenoses number 2, narrowing angle $32^{\circ}$.

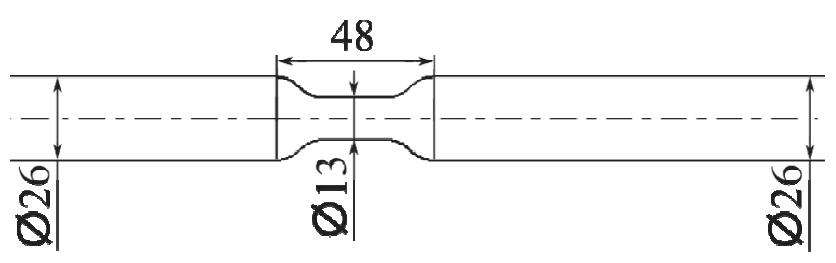

Figure 4. Symmetric stenoses number 3, narrowing angle $45^{\circ}$.

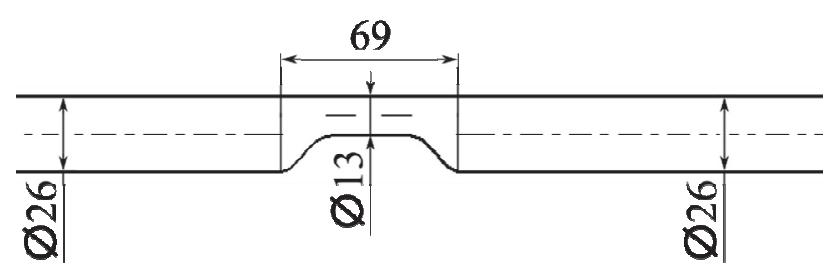

Figure 5. Eccentric stenoses with circular cross-section, narrowing angle $50^{\circ}$.

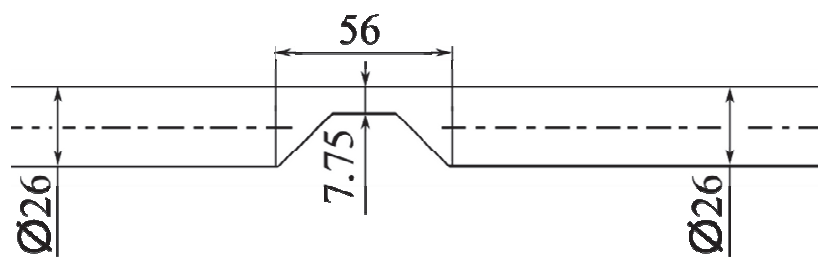

Laser optical measurement technique PIV (Particle Image Velocimetry) was employed for flow visualization and measurement. In this work, PIV technique was used for evaluation the velocity field past obstructed part of models.

The aims of this experimental study are twofold. Firstly see if static pressure loss was affected more by shape of stenosis or by eccentricity, secondly see how shape of stenosis and eccentricity affected velocity field behind stenosis.

\section{Methods}

\subsection{Pressure loss}

Schematic diagram of the experimental setup for measuring the pressure-flow is shown in Figure 7.

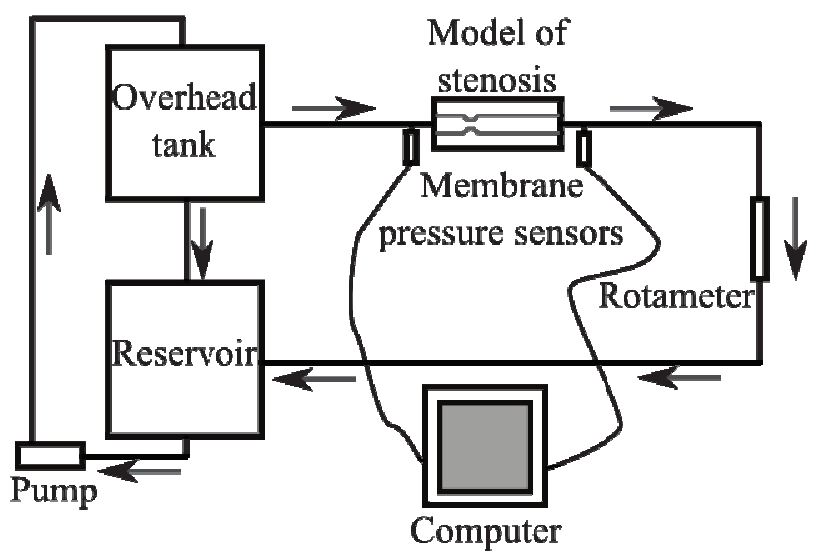

Figure 7. Experimental setup.

The steady flow was established in the models. The pressure was measured by membrane pressure sensors. Pressure was measured $16 \mathrm{~cm}$ upstream and $30 \mathrm{~cm}$ downstream of the sensors. The sensors were calibrated before measurement using water column.

The working fluid was water at room temperature. Flow rate was measured using a rotameter. The flow conditions approximate physiological flow in vessels, the measuring range of Reynolds number in the unobstructed part of model was from 130 to 2730 .

For each flow regime the pressure gradient between probes was recorded. The pressure loss coefficient for each models were calculated from obtained experimental data using Equation 3 by polynomial regression analysis.

\subsection{Visualization}

Schematic diagram of the experimental setup for visualization velocity field in models is same as setup on Figure 7. The measured area was illuminated by two continual lasers and CCD camera was employed for image acquisition. The camera was placed above the model and the model was illuminated by laser in axial plane.

Figure 6. Eccentric stenoses with non-circular cross-section, narrowing angle $45^{\circ}$. 
The four regimes of steady flow of water at room temperature were investigated by PIV method. The flow was seeded with fluorescent particles. The measuring range of Reynolds number in unobstructed part of model was from 130 to 818 ; the range corresponds to physiological flow in calf vessel.

For each regime 100 images were captured. The images were analysed by software Dantec Dynamics Studio.

\section{Results}

\subsection{Pressure loss}

Table 1 summarises the set of experimental results obtained for models of stenoses. In all experiment, correlation coefficient was higher then 0.95 .

Table 1 show that the local pressure loss coefficient increases with increasing narrowing angle of the symmetric stenoses. The highest value of local pressure loss coefficient of symmetric stenoses has stenosis with the highest value of narrowing angle.

Similarly the local pressure loss coefficient increases with changing of eccentricity. Eccentric stenoses have higher value of local pressure loss coefficient than symmetric stenoses. The maximum value of local loss coefficient has eccentric stenosis with non-circular crosssection.

Figure 8 shows relationship between the local pressure loss coefficient and angle of narrowing. Figure 8 shows that symmetric stenosis number 3 (c) and eccentric stenosis with non-circular section (e) have same narrowing angle, but value of local pressure loss coefficient is greater for eccentric stenosis.

Figure 9 plots relationship between local pressure loss and flow rate.

Considering the same boundary conditions, the pressure drop in a narrowed lumen causes flow reduction in comparison with an unobstructed diameter. Decrease of flow volume was expressed by the ratio $\mathrm{Q}_{\max } / \mathrm{Q} . \mathrm{Q}_{\max }$ is the flow rate in unobstructed tube, $\mathrm{Q}$ is actual flow rate for given shape of stenosis. For the ratio $Q_{\max } / \mathrm{Q}$ the equation can be applied:

$$
\frac{Q_{\max }}{Q}=\frac{1}{\sqrt{1+\xi}}
$$

The ratios of $\mathrm{Q}_{\max } / \mathrm{Q}$ for measured stenosis geometries are plotted in Figure 10. The flow rate changes from approximately $27 \%$ to $22 \%$ of maximum flow rate. The minimal decrease is reached for the smoothest symmetric geometry, the maximum drop in flow rate occurs for eccentric stenoses.

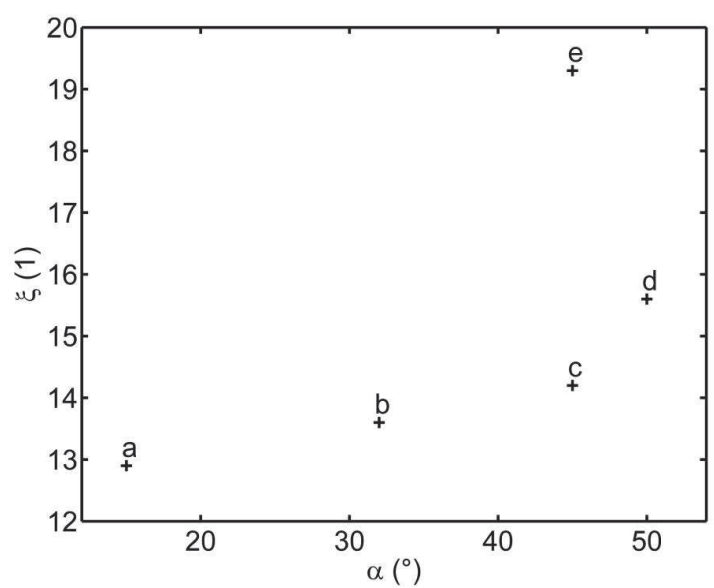

Figure 8. Relationship between the local loss coefficient and angle of narrowing; a - symmetric number $1, b$ - symmetric number $2, \mathrm{c}$ - symmetric number $3, \mathrm{~d}$ - eccentric with circular cross-section, e - eccentric with non-circular cross-section.

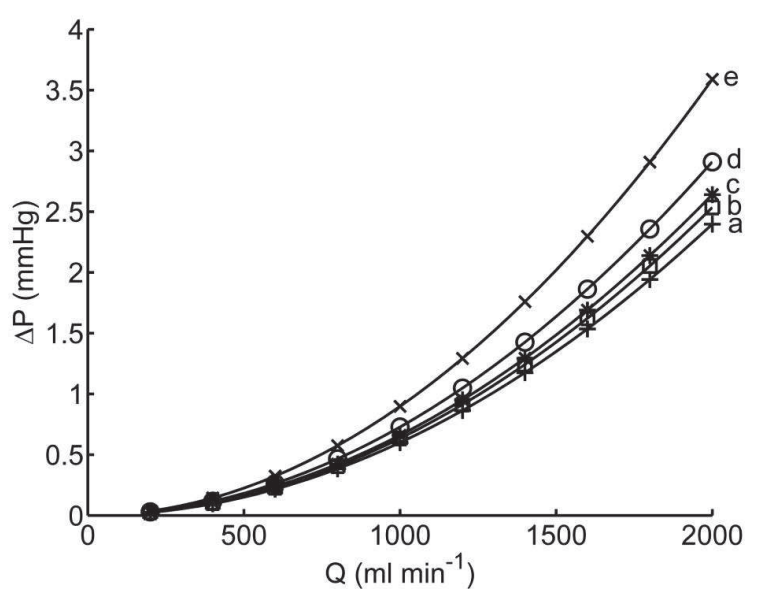

Figure 9. Relationship between the local loss pressure loss and flow rate; a-symmetric number $1, \mathrm{~b}$ - symmetric number $2, \mathrm{c}-$ symmetric number $3, \mathrm{~d}-$ eccentric with circular cross-section, $\mathrm{e}-$ eccentric with non-circular cross-section.

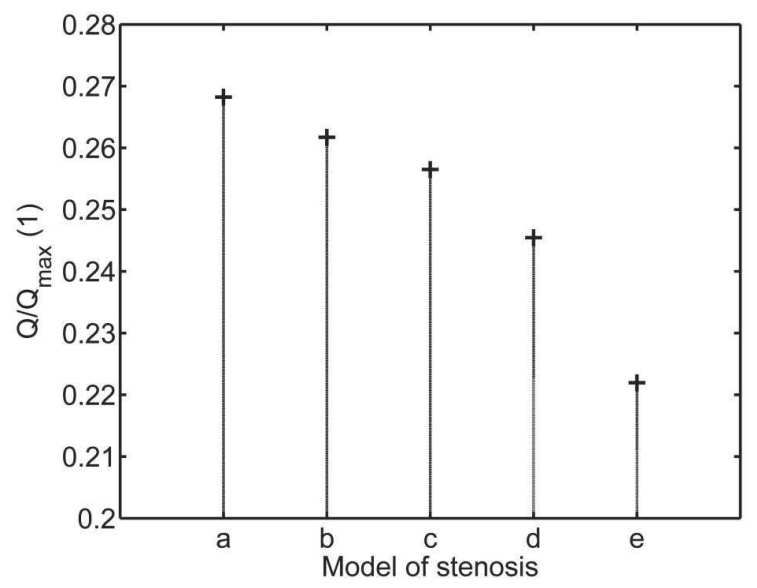

Figure 10. Flow rate drop of models; a - symmetric number 1 , $\mathrm{b}$ - symmetric number 2, c-symmetric number $3, \mathrm{~d}$ - eccentric with circular cross-section, e - eccentric with non-circular cross-section. 
Table 1. Local pressure loss coefficient for models of stenosis.

\begin{tabular}{|c|c|c|c|}
\hline Stenosis & Geometry of stenosis & Cross-section & Local pressure loss coefficient \\
\hline $\begin{array}{c}\text { Symmetric } \\
\text { number } 1 \text { (a) }\end{array}$ & & & 12.9 \\
\hline $\begin{array}{c}\text { Symmetric } \\
\text { number } 2 \text { (b) }\end{array}$ & & & 13.6 \\
\hline $\begin{array}{c}\text { Symmetric } \\
\text { number } 3 \text { (c) }\end{array}$ & & & 14.2 \\
\hline $\begin{array}{l}\text { Eccentric } \\
\text { circular (d) }\end{array}$ & --1 & & 15.6 \\
\hline $\begin{array}{c}\text { Eccentric } \\
\text { non-circular (e) }\end{array}$ & & & 19.3 \\
\hline
\end{tabular}

\subsection{Visualization}

The visualization and velocity measurement was performed in order to provide qualitative and quantitative data on the flow field behind stenosis. This is important since the degree of stenosis within arteries is often diagnosed from the maximum velocity measured by Doppler ultrasound system.

Figure 11 shows results for the lowest $\operatorname{Re}(\operatorname{Re}=130)$ and figure 12 shows results for the highest $\operatorname{Re}(\operatorname{Re}=818)$. The curves show the development of the maximum measured velocity in the axial plane behind stenosis. The maximum velocity $U_{\max }$ is normalized by maximum velocity in the stenosis. The $\mathrm{x} / \mathrm{D}$ ratio expresses the distance from the stenosis. The distance is given in multiples of unobstructed diameter D. Figure 13 and 14 show velocity field for $\operatorname{Re} 130$ and 818 for stenosis number 1 (a) and number 3 (c) in the form of streamlines.

\section{Conclusions}

In this work, experimental investigations of pressure loss coefficient for the five models of stenosis were performed: three symmetric stenoses with different shape and two eccentric stenoses with different crosssection. All models were stenosis of $75 \%$ area reduction. The results prove the relationship between pressure loss and varying eccentricity and shape of stenosis. The eccentric stenoses create higher pressure loss then the symmetric stenoses, the eccentric stenosis with noncircular cross-section create higher pressure loss then the eccentric stenosis with circular section.

Investigation of the relationship between pressure loss and geometry of stenoses indicates that static pressure loss coefficient is affected by the shape of stenosis, but it was affected more significantly by the eccentricity.

The results obtained by PIV method prove that the development of the velocity profile past stenosis depends on shape of stenosis and Reynolds number. This fact could be crucial for the determination of stenosis severity in clinical diagnosis.

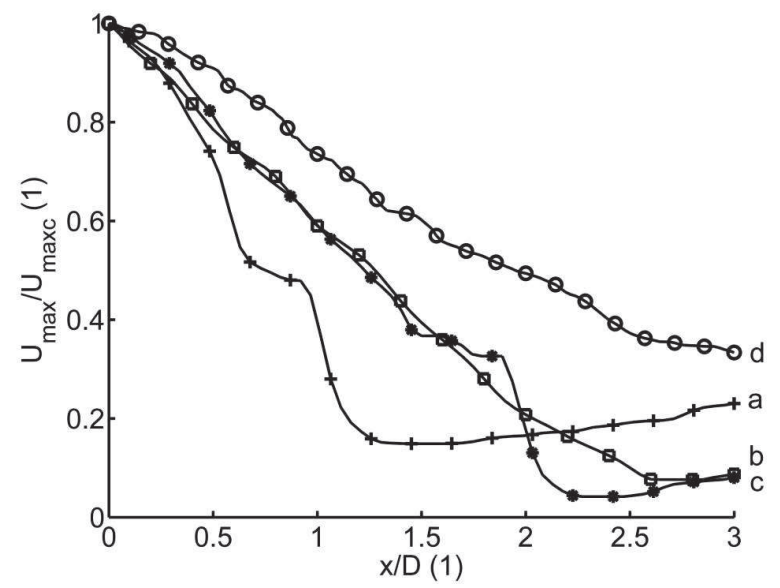

Figure 11. Maximum velocity curve for $\mathrm{Re}=130$; a - symmetric number $1, \mathrm{~b}$ - symmetric number 2 , c symmetric number $3, \mathrm{~d}-$ eccentric with circular cross-section.

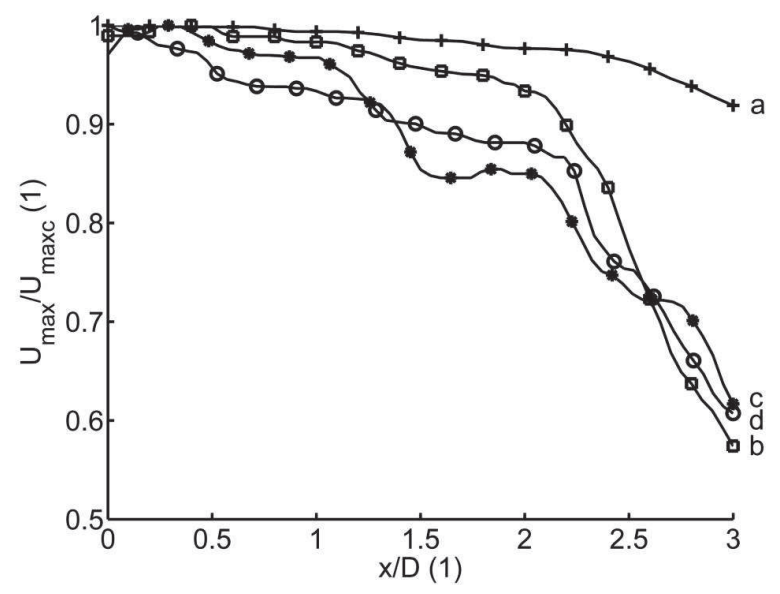

Figure 12. Maximum velocity curve for $\mathrm{Re}=818$; a - symmetric number $1, \mathrm{~b}$ - symmetric number 2 , c symmetric number $3, \mathrm{~d}-$ eccentric with circular cross-section. 


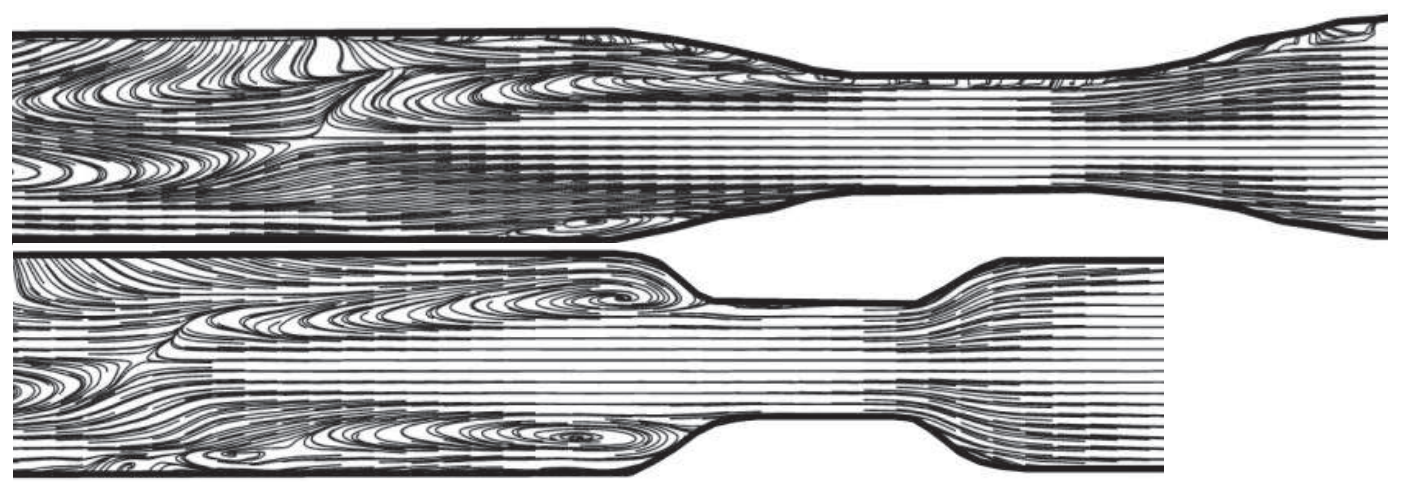

Figure 13. Velocity field for $\mathrm{Re}=130$ for stenosis number 1 (a) and for stenosis number 3 (c).

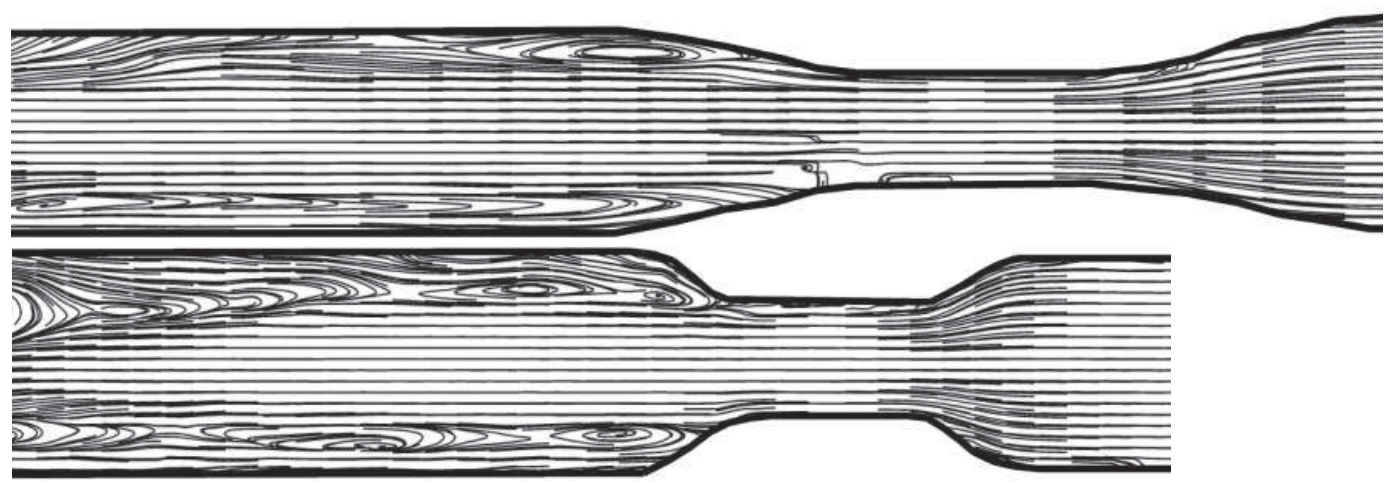

Figure 14. Velocity field for $\mathrm{Re}=818$ for stenosis number 1 (a) and for stenosis number 3 (c).

\section{References}

1. L. Caplain, A 70-year-old man with a transient ischemic attack, J Am Med Association, 300, 81 - 90 (2008)

2. S. Glagov, C. K. Zarins, D. P. Giddens, D. N. Ku, Hemodynamics in atherosclerosis: insights and perspective gained from studies of human arteries, Arch. Pathol. Lab. Med., 316, 1371 - 1375 (1988)

3. Y. Jiang, K. Kohara, K. Hiwada, Low wall shear stress in carotid arteries in subject with left ventricular hypertrophy, Am Jounar of Hypertension, 13 (8), $892-898$ (2000)

4. C. G. Caro, Discovery of the Role of Wall Shear in Atherosclerosis, Arteriscler Thromb Vasc Biol., 29, $158-161$ (2009)

5. T. M. Liou, S. N. Liou, Review on In Vitro Studies of Hemodynamics Characteristics in Terminal and Lateral Aneurysm Models (Invited Review Paper), In Proc. Natl. Sci. Counc. ROC(B), 23 (4), $133-148$ (1999)

6. D. N. Ku, Blood Flow in Arteries, Ann Rev Fluid Mech., 29, 399 - 434 (1997)

7. A. S. Shuib, P. R. Hoskins, W. J. Easson, Flow Visualization and Characterization of an Artery Model with Stenosis, Engineering and Technology, 60, 56 - 69 (2011)

8. B. Liu, The influence of stenosis on the downstream flow pattern in curved arteries, Medical Engineering and Physics, 29, 868 - 876 (2007)
9. A. S. Roy, L. H. Back, R. K. Banerjee, Guidewire flow obstruction effect on pressure drop-flow relationship in moderate coronary artery stenosis, Journal of Biomechanics, 39, 853 - 864 (2006)

10. K. Pielhop, M. Klaas, W. Scroder, Analysis of the unsteady flow in a elastic stenotic vessel, European Journal of Mechanics of Fluid, 35, 102 - 110 (2012)

11. J. Kolínský, J. Matěcha, J. Novotný, PIV Measurement of Oscillatory Flow Field in Compliant Tube, In Proc. Of ASME 2009 Fluids Engineering Division, New York, (2009)

12. D. Young, Fluid mechanics of arterial stenosis., $J$ Biomech Engineering, 101, 185 - 96 (1979)

13. L. A. Heflin, C. B. Street, D. V. Papavassilio, E. A. O'Rear, A computational investigation of the geometry factors affecting the severity of renal arterial stenosis, J Biorheol, 23, 102 - 110 (2009)

14. D. Tang, Ch. Yang, S. Kobayashi, J. Zheng, R. P. Vito, Effect of stenosis asymmetric of blood flow and artery compr.: A three-dim. fluid-struc. interaction model, Annals of Biomedical Engineering, 31, 1182 - 1193 (2003)

15. S. R. Doods, The haemodynamics of asymmymetric stenosis, Eur J Vasc Surg, 24, 332 - 337 (2002) 